\title{
Increased luminescence efficiency by synergistic exploitation of lipo/hydrophilic co-solvency and supramolecular design $\dagger$
}

Giulia Tregnago $^{1 \S}$, Michele Serri ${ }^{2,8 \S}$, Sergio Brovelli ${ }^{3}$, Shane O. McDonnell ${ }^{4}$, Platon

Korniychuk ${ }^{5}$, Linjun Wang ${ }^{6}$, Michael Wykes ${ }^{7}$, David Beljonne ${ }^{7}$, Adam Tracz ${ }^{5}$, Harry L.

Anderson $^{4}$, Franco Cacialli ${ }^{1 *}$

${ }^{I}$ Department of Physics and Astronomy and London Centre for Nanotechnology

University College London, London WC1E 6BT, United Kingdom

${ }^{2}$ Department of Materials, Imperial College London, London SW7 2AZ, United Kingdom

${ }^{3}$ Department of Materials Science, University of Milano-Bicocca, Milano I-20125, Italy

${ }^{4}$ Department of Chemistry, University of Oxford, Oxford OX1 3TA, United Kingdom

${ }^{5}$ Center of Molecular and Macromolecular Studies, Lodz 90363, Poland

${ }^{6}$ Department of Chemistry, Zhejiang University, Hangzhou 310027, China

${ }^{7}$ Laboratory for Chemistry of Novel Materials, University of Mons, Mons 7000, Belgium

${ }^{8}$ Department of Chemistry "U. Schiff”" \& INSTM RU Firenze, Università di Firenze, Sesto

Fiorentino 50019, Italy

${ }^{\S}$ These authors contributed equally to this work

*e-mail: f.cacialli@ucl.ac.uk

$\uparrow$ Electronic supplementary information (ESI) available: experimental and computational details, absorption spectra analysis, normalized time-resolved emission spectra, time dependence of the average emission energy, and molecular modeling and simulations. 


\section{Abstract}

We use steady-state and time-resolved photoluminescence (PL) spectroscopy to investigate the luminescent properties of a sulfonated poly(diphenylenevinylene) lithium salt (PDV.Li) in water/propanol solutions at different concentrations, with a view to assessing its aggregation behavior. In particular, we compare results from uninsulated PDV.Li and cyclodextrin-threaded PDV.Li polyrotaxane (PDV.LiC $\subset-C D)$. We find that addition of 1propanol ( $\geq 20$ weight $\%$ ) leads to a significant blue-shift (of $\sim 0.20 \mathrm{eV}$ ) of the PL spectra, that we assign to suppressed interchain aggregation in PDV.Li solutions, with a concomitant fourfold increase in the fluorescence quantum efficiency (i.e. from 14 to $60 \%$ ). Surprisingly, a moderate concentration of propanol increases further the luminescence efficiency even for PDV.Li $\subset \beta-C D$, whose supramolecular encapsulation already provides a shield against aggregation. Indeed, addition of propanol reduces the solvent polarity, and therefore helps solubilizing these materials that are still largely aromatic in nature. Interestingly, however, both uninsulated PDV.Li and polyrotaxane solutions exhibit signs of aggregation at high propanol fraction $(>70 \%)$ with a distinctively weaker coupling than that of interchain states in PDV.Li at high water concentration and in pure water in particular. While we ascribe such behavior to a poor solvation of the polar moieties, we also report a different strength of aggregation for PDV.Li and PDV.LiC $\subset-C D$ that can be attributed to the presence of the cyclodextrin rings. In PDV.Li $\subset \beta-\mathrm{CD}$ hydrogen bonding between the cyclodextrin rings may lead to closer packing between the polymer chains. We therefore suggest that a content of propanol between 30 and $70 \%$ provides a good balance of hydrophobic and hydrophilic interactions both for PDV.Li and PDV.LiC $\beta-C D$.

\section{Introduction}


Conjugated polyelectrolytes (CPEs) have attracted a growing interest in the past decade due to their unique set of properties. In the first instance, CPEs preserve the electronic properties and photoluminescence of their $\pi$-conjugated core, which can all be widely exploited in optoelectronic devices. At the same time the presence of ionic groups ensure good solubility in environmentally-friendly and mainly water-based solvents, ${ }^{1,2}$ and make them suitable for use in combination with ionic liquids. ${ }^{3}$ The delocalization and migration of excitons along the conjugated backbone also causes the so-called "amplified (fluorescence) quenching" by oppositely-charged ionic quenchers in solution, which has been exploited in fluorescence-based chemo- and bio-sensors. ${ }^{4-6}$

The photophysics of these systems is strongly influenced by the coexistence of ionic groups and apolar moieties along the CPE backbones, which yields a complex interplay of Coulombic and hydrophobic interactions between the polymer chains in solution. The photophysical properties of these systems are therefore strongly dependent on the solvent(s) used, also in relation to the potential formation of a variety of ordered supramolecular nanostructures spanning from random coils, to helical conformations. ${ }^{7,8}$ In polar solvents, in particular, hydrophobic and $\pi-\pi$ interactions between the conjugated (aromatic) moieties provide a tendency to aggregation of the conjugated backbones even in diluted solutions, thereby leading to the formation of interchain excited states. In particular, intermolecular interactions in the ground state lead to the formation of aggregates whereas if a polymer chain (or rather a segment) in the excited state interacts attractively with another chain (and their interaction in the ground state is repulsive) the resulting state is an excimer. Both species act as exciton traps and usually yield a reduction in photoluminescence quantum efficiency (PLQE) together with clear changes in the absorption and photoluminescence (PL) emission spectrum. A strategy for suppressing such detrimental effects on fluorescence is to control the interchain spacing by functionalization of the conjugated backbone with bulky 
substituents, or by supramolecular encapsulation of the polymer chains in optically inert macromolecules such as polysaccharides and polyethers. ${ }^{1,9,10}$ In previous work we have shown that threading cyclodextrin macrocycles on a conjugated polymer backbone to form polyrotaxanes can achieve efficient reduction of interchain species formation and interchain exciton migration in solution and in thin films. ${ }^{11-14}$

An alternative approach to suppress the formation of interchain states is the use of a co-solvent system. In the pharmaceutical field, in particular, co-solvency is a well-established and successful technique to increase drugs solubility. ${ }^{15}$ Indeed, CPEs are generally soluble in water/alcohol mixtures at all concentrations, and it was reported that in the case of a sulfonated poly(phenylene ethynylene) in water/methanol solutions reducing the solvent polarity by increasing the alcohol fraction also hinders the formation of interchain species. ${ }^{4,16}$

In this work we investigate, by means of continuous wave $(\mathrm{CW})$ and time-resolved PL spectroscopy combined with molecular modeling, the effect of the alcohol 1-propanol on the aggregation of sulfonate substituted poly(diphenylene-vinylene) lithium salt (PDV.Li, see Figure 1), and its polyrotaxane derivative (PDV.LiC $\subset$-CD) in water/1-propanol mixtures (with 1-propanol fractions ranging from $0 \%$ to $99.5 \%$ ).

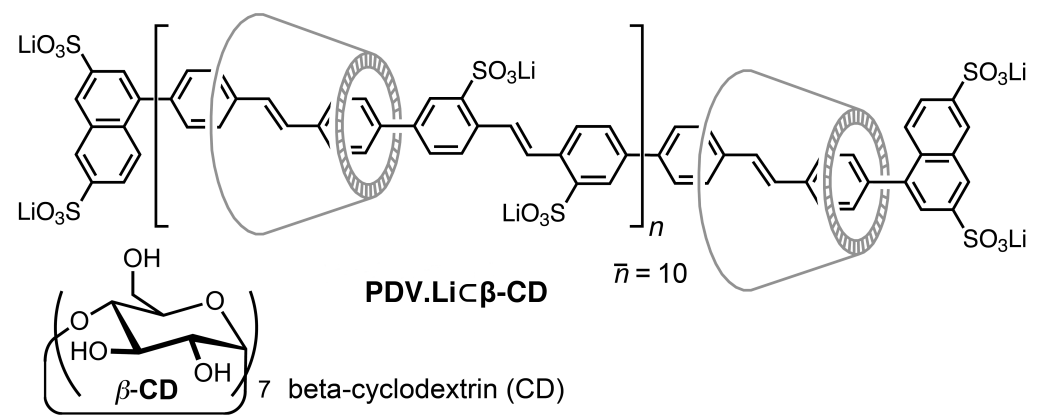

Figure 1 Chemical structures of the sulfonate substituted poly(diphenylene-vinylene) lithium salt rotaxane (PDV.LiC $\angle$-CD) and cyclodextrin ( $\beta-\mathrm{CD})$ macrocycle. PDV.Li is the same as PDV.LiC $\_-\mathrm{CD}$ except without the threaded cyclodextrin rings.

The photophysics of PDV.Li and PDV.LiC $\beta-\mathrm{CD}$ in pure water solutions has already been reported. ${ }^{14}$ Significant aggregation was observed even in relatively diluted solutions for the PDV.Li, but was nearly completely suppressed for the polyrotaxanes analogues in which 
the cyclodextrin shielding well preserved the intramolecular exciton dynamics. Here, we find that water and 1-propanol form a co-solvent system that significantly destabilizes the formation of interchain states in solutions of unthreaded and threaded PDV.Li, with a more pronounced effect in the unthreaded polymer. Interestingly, when the alcohol fraction is increased above $70 \%$, the temporal PL decays become markedly non mono-exponential. This suggests the onset of aggregation both in PDV.Li and PDV.LiC $\subset-C D$ albeit with a weaker coupling than that of interchain states formed in PDV.Li water solution that yielded a more significant reduction of the PL efficiency.

A molecular modeling study was carried out to complement the detailed spectroscopic investigation. Atomistic simulations of model aggregates provide insight into the interchain packing distances in PDV.Li and PDV.LiC $\subset$-CD and characterization of their excited states. Conclusions drawn from the experimental photophysical investigation are further supported by spectra simulated using phenomenological models of single chains and H-aggregates. Unthreaded PDV.Li in water forms strongly-coupled aggregates with predominant H-like character characterized by a partial charge transfer character. When both propanol and water are present in significant amounts (1-propanol fraction $<70 \%$ ) the PL is consistent with isolated emitters, while at high propanol fractions $(>70 \%)$ we believe that the majority of polymer chains forms weakly-coupled H-like aggregates. A different behavior is observed in the polyrotaxane analogue in propanol-rich solutions, where the alcohol seems to induce stronger excitonic coupling than in PDV.Li. In fact, by varying the propanol content, we observe the formation of interchain aggregates with excitonic couplings $(J)$ ranging from 18 $\mathrm{meV}$ to $100 \mathrm{meV}$. As discussed by Spano, the excitonic coupling is considered weak when the nuclear relaxation energy (typically $\sim 0.18 \mathrm{eV}$ for conjugated polymers) is greater than the exciton bandwidth (that is, $\sim 4 J$ in a tight-binding approximation as applied to a one- 
dimensional stack $)^{17,18}$. Therefore when $J$ is lower than $\sim 45 \mathrm{meV}$, the system is treated within the weak excitonic coupling regime.

\section{Results and Discussion}

\subsection{Spectral characteristics in water/propanol mixtures}

The series of CW absorbance and PL measurements, performed on the PDV.Li and PDV.LiC $\beta-C D$ solutions in water/propanol mixtures with different propanol content, is shown in Figure $2 \mathrm{a}$ and $2 \mathrm{~b}$, respectively. The absorption spectra of both threaded and unthreaded PDV.Li have the typical broad and featureless profile of conjugated polymers in solution. We do observe minor effects of the solvent composition on the absorption spectrum: (i) a weak blue-shift (Figure S1a) that increases as the water content decreases and that could be due to either changes in solvent polarity or to the formation of $\mathrm{H}$-like aggregates, or both; (ii) and small changes in the linewidth of the spectrum (Figure S1b). In particular, we note that both for PDV.Li and PDV.LiC $\beta-C D$ the linewidth reaches its minimum for $1: 1$ water:propanol solutions (0.59 and $0.53 \mathrm{eV}$ for PDV.Li and PDV.Lic $\beta-\mathrm{CD}$, respectively), whereas it increases for lower and higher content of propanol. We note that although in theory the absorption spectrum can be indicative of interchain species formation and discriminate between aggregates and excimer, with aggregates in principle showing some features in absorption spectrum and excimers not, this is, however, a rather theoretical possibility for most luminescent polymers, with very few notable exceptions, such as poly(3hexylthiophene) (P3HT). ${ }^{19,20}$ The absorption spectrum is the convolution of different species, and, especially where the concentration is expected to be low, the related aggregate band could be masked by the absorption of the non (or less) aggregated species. Furthemore, a distinctive feature of emission with respect to absorption is its selectivity. Spectral diffusion during the excited states lifetime leads the excitons to localise on the lowest energy sites, and 
so one loses the contribution (to PL) that would arise from the higher energy gap (i.e. nonaggregated) sites, thereby allowing detection of interchain states (in PL), even when their concentration makes them virtually undetectable in the absorption spectrum. Such states have a distinctive fluorescence decay time, that in the case of excimer-like states is a factor of $\sim 100$ higher than that of the "isolated" molecule ${ }^{21,22}$ therefore one would expect much longer lifetimes rather than few nanoseconds as found in the manuscript (see below). We also notice that PDV.Li and its cyclodextrin-threaded form present remarkably similar absorption spectra, thereby suggesting that the encapsulation does not perturb the electronic properties of the conjugated cores. This is consistent with calculations showing that the inner cavity of the cyclodextrins is large enough to accommodate the conjugated cores without placing conformational constraints on the core themselves. ${ }^{23}$ 

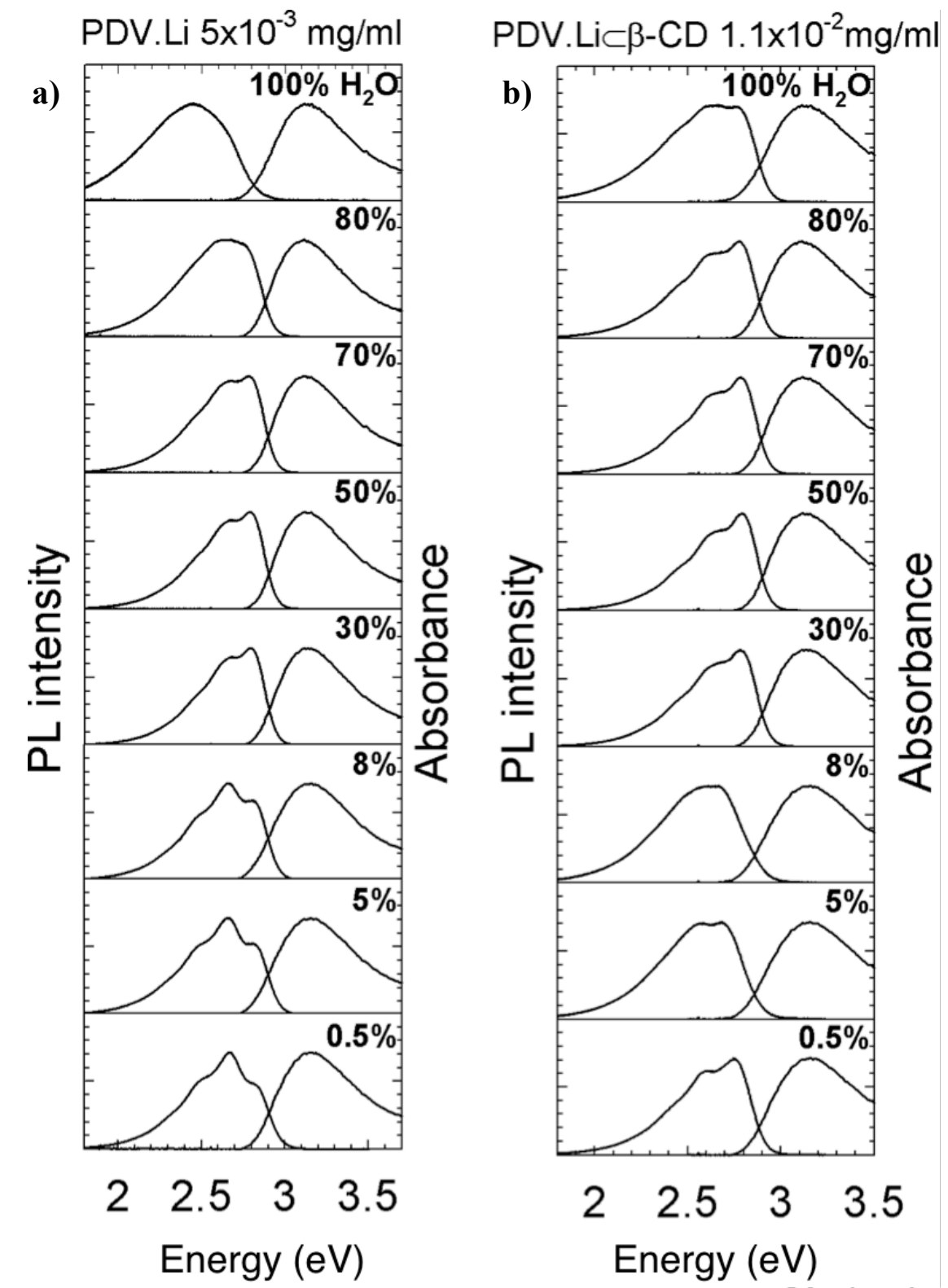

Figure 2 Emission and absorption spectra of PDV.Li (a) and PDV.LiC $\subset-C D$ (b) solutions in water/propanol mixtures (\% water is indicated). All data shown were taken for PDV.Li and PDV.LiC $\beta-C D$ solutions in water/propanol mixtures at a concentration of $5 \times 10^{-3} \mathrm{mg} \cdot \mathrm{mL}^{-1}$ and $1.1 \times 10^{-2} \mathrm{mg} \cdot \mathrm{mL}^{-1}$ respectively, in order to account for different molecular weights and obtain the same optical density.

By looking at the fluorescence of solutions in water (Figure 2a), we find that the unthreaded polymer PDV.Li exhibits a broad, featureless emission spectrum $(\mathrm{FWHM}=0.60 \mathrm{eV})$ with a maximum at $2.44 \mathrm{eV}$ (although we note that in the normalised time-resolved emission spectra in Figure S2a the PL spectrum of PDV.Li is not totally structureless). Compared to PDV.LiC $\beta$-CD (Figure 2b), the emission peak of PDV.Li is shifted by about $0.22 \mathrm{eV}$ towards the red and the fluorescence quantum efficiency is 
significantly lower (PLQE $=14 \pm 1 \%$ in PDV.Li against $33 \pm 3 \%$ in PDV.LiC $\subset-C D$ ), as shown in Figure 3. As the propanol fraction is increased from $0 \%$ to $50 \%$, the PL spectrum of PDV.Li solutions evolves to a narrower $(\mathrm{FWHM}=0.43 \mathrm{eV})$ and blue-shifted emission profile characterized by higher PLQE. The solution in the 70:30 water/propanol mixture already shows PL with a resolved structure, characterized by a vibronic progression with $\mathrm{E}_{00}=2.78 \mathrm{eV}$ and a vibrational energy of $0.17 \mathrm{eV}$ associated with the $\mathrm{C}=\mathrm{C}$ stretching mode coupled to the electronic transition ${ }^{18}$. When the propanol fraction increases to $50 \%$, the emission from the 0-0 band clearly dominates and the PLQE reaches its highest value $(60 \pm 6 \%)$. PDV.LiC $\beta$-CD polyrotaxanes show analogous, yet less pronounced trends in PLQE, with an increase from $33 \pm 3 \%$ to $55 \pm 6 \%$, and a narrowing of the PL spectrum FWHM from $0.55 \mathrm{eV}$ to $0.39 \mathrm{eV}$ as the propanol fraction is increased from $0 \%$ to $50 \%$.

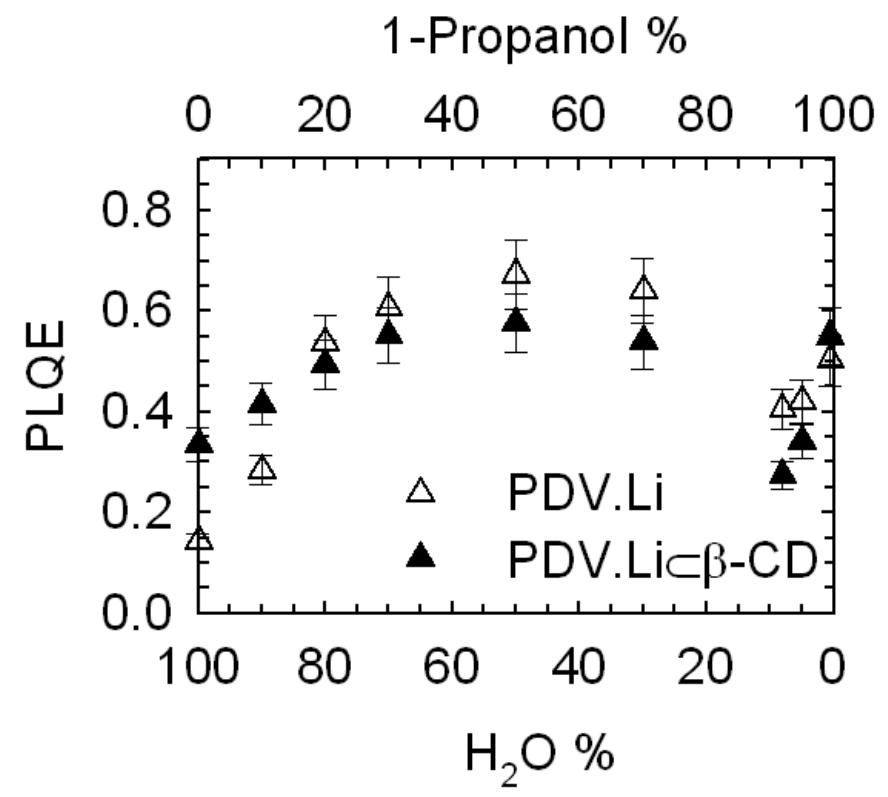

Figure 3 Fluorescence quantum yields of PDV.Li and PDV.LiC $\beta$-CD solutions.

Following further increments of the propanol fraction $(>70 \%)$, we assist to a change of the PL spectrum profile of PDV.Li characterized by the increase in intensity of the 0-1 vibronic component centered at $2.6 \mathrm{eV}$ (Figure $2 \mathrm{a}$ ), and a reduction of the PLQE to $40 \pm 4 \%$ (Figure 3). Similarly, the polyrotaxane solutions experience a drop in the PLQE to $30 \pm 3 \%$, a 
broadening of the PL spectrum (FWHM $=0.50 \mathrm{eV}$ at propanol 95\%) and an increase of the 0 1 band (Figure $2 b$ ) although less pronounced compared to PDV.Li.

Surprisingly, at very high propanol content (99.5\%), the PLQE of both the polyrotaxane and the PDV.Li solutions revert to about 55\%. Also, a concomitant recovery of the intensity of the 0-0 band is observed in the PDV.LiC $\beta-C D$ PL spectrum.

The characterization of the steady-state PL of PDV.Li and PDV.LiC $\beta$-CD solutions was complemented by extensive investigation of the time dependence of the PL emission. In Figure 4 we show the contour plots of the PL intensity as a function of time and photon energy whereas in Figure 5 we report the decays of the PL intensity. Looking first at PDV.Li solutions in pure water (Figure 4a) we note a comparatively fast decay in the region above $2.75 \mathrm{eV}$, whereas below $2.6 \mathrm{eV}$ we note the presence of a band whose emission spans over several nanoseconds. The PL decay at $2.75 \mathrm{eV}$ is strongly non mono-exponential (Figure 5a) and fitting to a bi-exponential law (Equation 1) allowed us to identify two main components with characteristic lifetimes $\tau$ of 680 ps (relative weight $=69 \%$ ) and $2.45 \mathrm{~ns}$ (relative weight $=31 \%)$, respectively.

$$
I(\mathrm{t})=A_{1} \exp \left(-\mathrm{t} / \tau_{1}\right)+A_{2} \exp \left(-\mathrm{t} / \tau_{2}\right) \quad \text { Equation } 1
$$

The population of these long-lived states is effectively reduced as propanol is added to the solution, while a band at $2.75 \mathrm{eV}$ emerges (Figure 4a and S2a). The decay at $2.75 \mathrm{eV}$ in solutions with $>30 \%$ propanol is single-exponential $(\tau=840 \mathrm{ps})$ over 3 intensity decades (Figure $4 \mathrm{a}$ for the $50 \%$ concentration).

When propanol is the main component of the solvent mixture (namely propanol fractions $92 \%, 95 \%, 99.5 \%$ ), we observe in PDV.Li a slight faster (from $\tau \sim 840$ ps to $\tau \sim 770$ ps) of the decay at $2.75 \mathrm{eV}$, which remains essentially mono-exponential. For energies below $2.6 \mathrm{eV}$, the decay becomes bi-exponential indicating a contribution from long-lived states, most evident in the solution containing 95\% propanol (Figure 5a). The PL emission in the 
first few ns after the excitation (Figure 4a and S2a) already shows the spectral changes observed in the CW spectra, consisting of a shift of the intensity maximum from $2.75 \mathrm{eV}$ to $2.6 \mathrm{eV}$. At longer delay times, the residual emission originates from a featureless band below $2.5 \mathrm{eV}$, similar to the one observed in pure water.
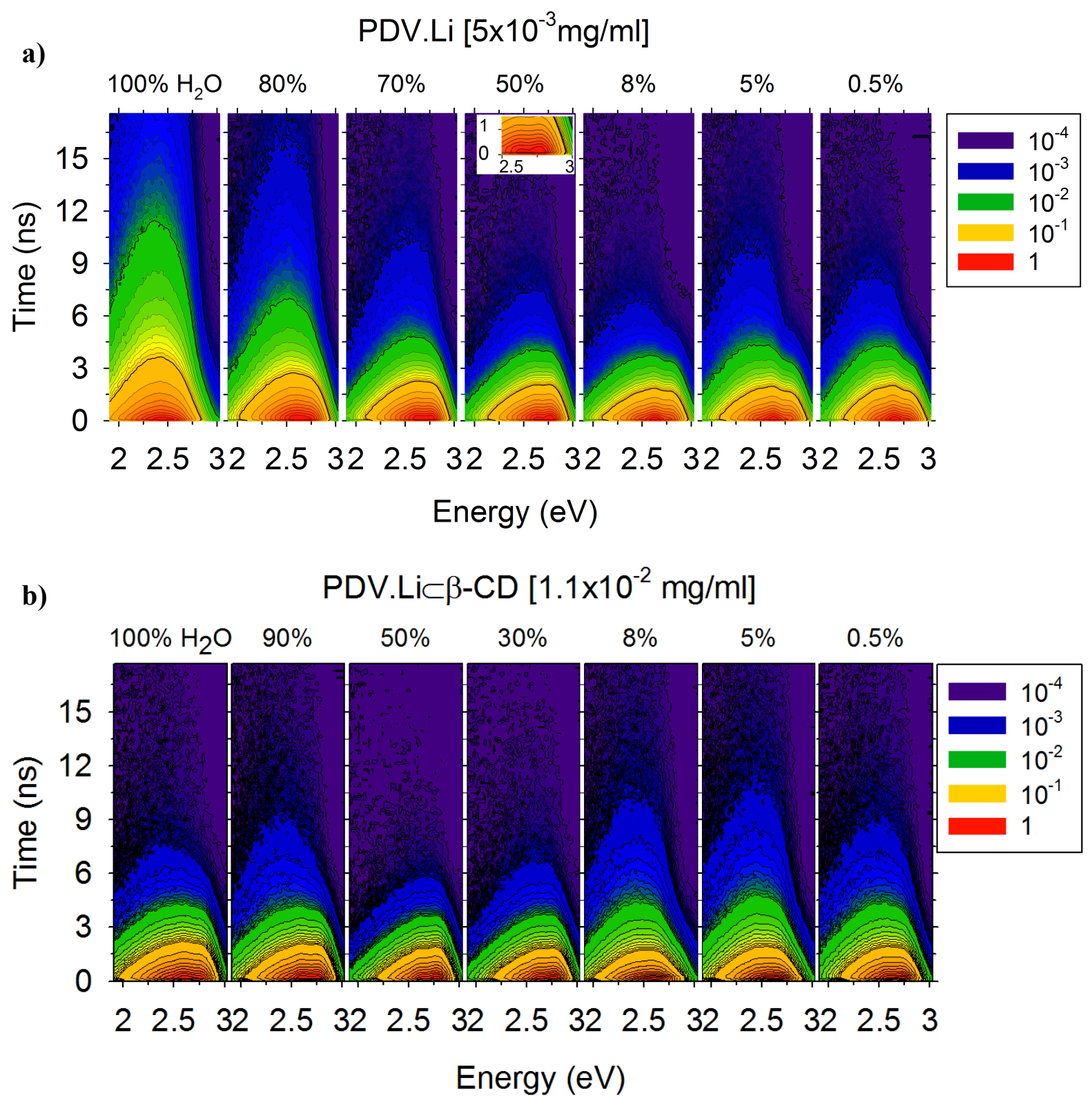

Figure 4 Contour plot of time-resolved emission spectra of PDV.Li (a) and PDV.LiC $\beta$-CD (b) solutions in water/propanol mixtures (\% water is indicated). For PDV.Li solution with 50\% propanol (a) we also show in the inset the evolution of the emission band at $2.75 \mathrm{eV}$ in the first $1.5 \mathrm{~ns}$ after excitation.

In contrast to PDV.Li, we do not observe in the polyrotaxane solutions such dramatic changes in the PL decay dynamics when propanol is added to solutions in water. The contour 
plots of the time-resolved emission spectrum of PDV.LiC $\beta-C D$ in water (Figure $4 \mathrm{~b}$ ) shows approximately energy independent decay dynamics. Only a moderate contribution to the PL from a long-lived band can be seen below $2.5 \mathrm{eV}$, which disappears completely when the propanol fraction is increased to 50\%. All solutions containing less than 50\% propanol display a PL decay at the emission maximum $(2.75 \mathrm{eV})$ characterized by single-exponential decay over two and a half intensity decades (Figure 5b) with excitation lifetimes varying between $\tau \sim 860 \mathrm{ps}$ in water and $\tau \sim 750 \mathrm{ps}$ in 1:1 water/propanol mixtures.

At high propanol fractions (92\% and 95\%), the time dependence of the PL decay for PDV.Li $\subset \beta$-CD polyrotaxane becomes energy dependent and clearly non mono-exponential (Figure 5b). The contour plots of time-resolved PL intensity (Figure 4b) and the normalized time-resolved emission spectra (Figure S2b) show exciton migration occurring in the first 3 ns after the excitation, associated with an acceleration of the decay at $2.75 \mathrm{eV}$ and the presence of long-lived emission $(\tau \sim 2 \mathrm{~ns}$ ) below $2.5 \mathrm{eV}$. Interestingly, at the highest propanol fraction considered $(99.5 \%)$ we observe a reduction of the population of long-lived emitting species and a partial recovery of the emission lifetime at $2.75 \mathrm{eV}$.

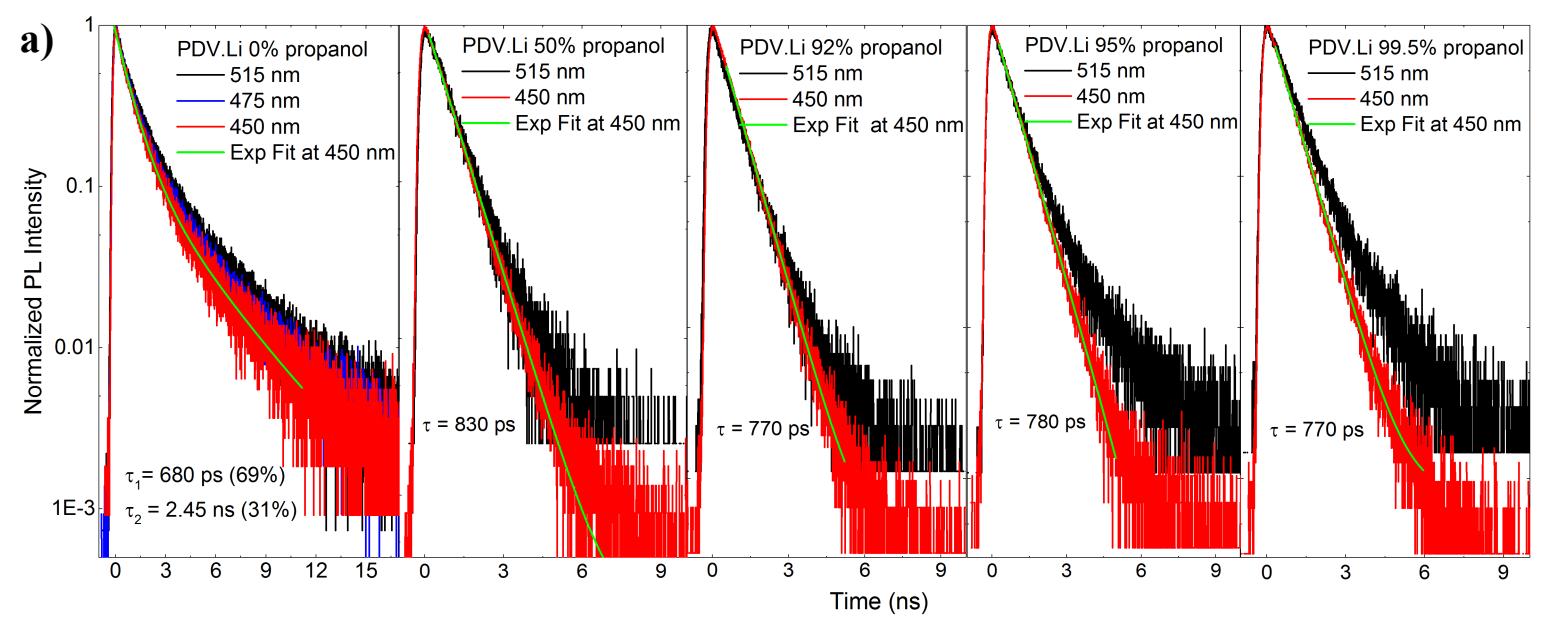




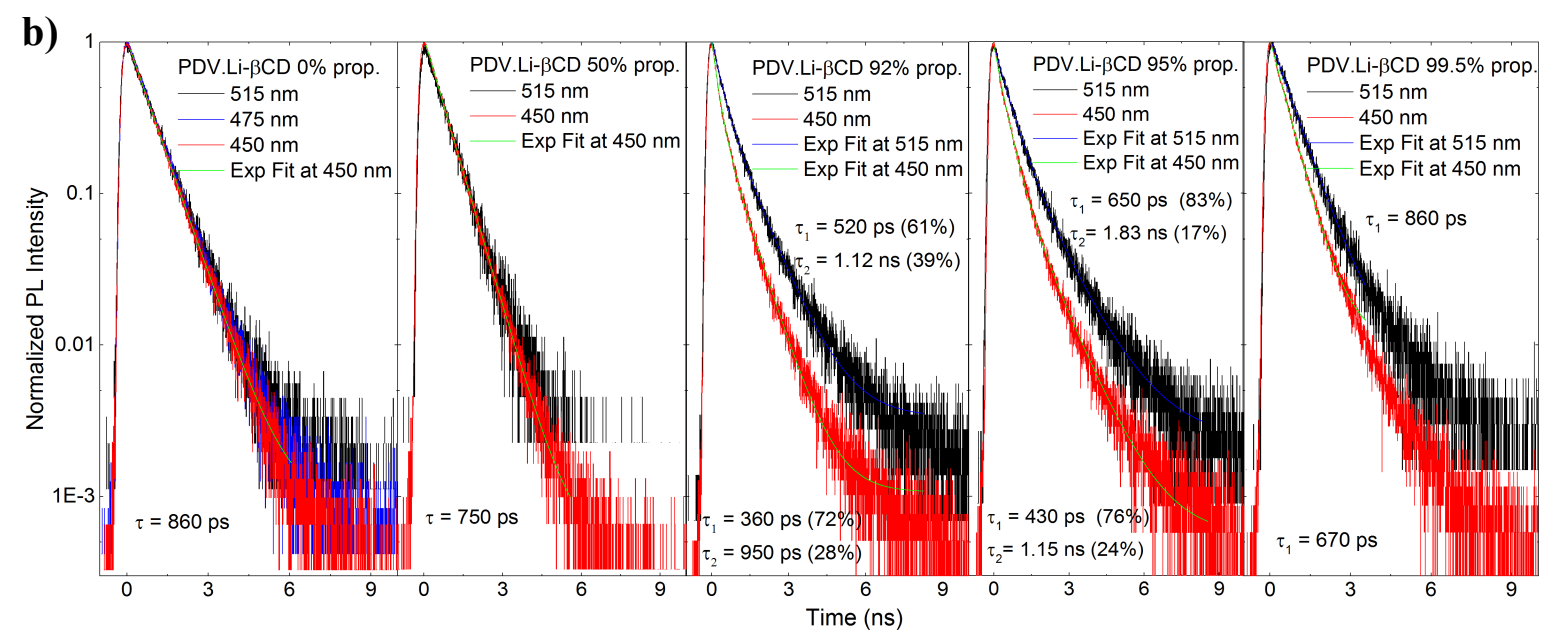

Figure 5 Decay of the fluorescence intensity in PDV.Li (a) and PDV.LiC $\beta-C D$ (b) solutions in water, $50 \%$ propanol, $92 \%$ propanol, $95 \%$ propanol and $99.5 \%$ propanol taken at different wavelengths.

\subsection{Chromophore aggregation in water solutions}

Our spectroscopic study of PDV.Li and PDV.LiC $\beta-C D$ solutions based on CW and time-resolved fluorescence measurements highlights, as expected, the profound modifications of the polymer photophysics induced by the cyclodextrin encapsulation and, more interestingly and rather unexpectedly, by changes in the composition of the water/propanol solvent mixture.

The spectroscopy of water solutions of PDV.Li and its polyrotaxane was the topic of a previous communication by our group. ${ }^{14}$ It was established that, in extremely diluted solutions $\left(10^{-4} \mathrm{mg} \cdot \mathrm{mL}^{-1}\right)$, both compounds exist as isolated molecules and have comparable photophysical properties. The onset of chromophore aggregation in water was observed in PDV.Li solutions as the concentration was increased by an order of magnitude $\left(10^{-3} \mathrm{mg} \cdot \mathrm{mL}^{-1}\right)$, while the fluorescence of PDV.LiC $\beta-\mathrm{CD}$ was rather insensitive to concentration thanks to the molecular insulation provided by the cyclodextrin rings. 
The solutions used in this work are moderately diluted $\left(5 \times 10^{-3} \mathrm{mg} \cdot \mathrm{mL}^{-1}\right.$ and $1.1 \times 10^{-2}$ $\mathrm{mg} \cdot \mathrm{mL}^{-1}$ for PDV.Li and PDV.LiC $\beta-\mathrm{CD}$ respectively, to ensure the same chromophore concentration) and, in agreement with the previous work, we found evidence of interchain states in PDV.Li chains in water and weak intermolecular interactions in the polyrotaxane. The unthreaded PDV.Li polymer exhibits a broad and featureless emission and much lower PLQE $(14 \pm 1 \%)$ in water compared to the polyrotaxane. We attribute these observations to a greater migration of the excitation energy from intrachain states to interchain states at lower energy with weak oscillator strength. This interpretation is consistent with the multi-exponential decay observed at $2.75 \mathrm{eV}$ (Figure 5a) that shows approximately two thirds of the excitons decaying with sub-ns dynamics $(\tau \sim 680 \mathrm{ps})$, typical of intrachain processes, ${ }^{14}$ and the remaining one third exhibiting an average lifetime of $2.47 \mathrm{~ns}$, due to a reduction of the radiative rate in the interchain states. On the other hand, the fluorescence of the polyrotaxane solutions in water decays single exponentially over 2 intensity decades between $2.75 \mathrm{eV}$ and $2.4 \mathrm{eV}(\tau \sim 860 \mathrm{ps}$, Figure $5 \mathrm{~b})$, suggesting that there is virtually only one emitting species.

\subsection{Inhibition of interchain states formation in the water/propanol co-solvent system}

As the propanol fraction is increased to $50 \%$, we observe that both solutions of PDV.Li and its polyrotaxane exhibit the characteristic emission of isolated chromophores. This is clearly seen in the single exponential decays of the emission, virtually independent from the energy of the emitted photons, which have excitation lifetimes $\left(\tau_{\mathrm{PDV} . \mathrm{Li}}=840 \mathrm{ps}\right.$, $\left.\tau_{\mathrm{PDV} . \mathrm{Li} \beta \mathrm{\beta CD}}=750 \mathrm{ps}\right)$ comparable with those measured in highly diluted solutions in water. The emission spectra of both the threaded and the unthreaded polymers distinctly show a vibronic progression, where three bands at $2.78 \mathrm{eV}, 2.61 \mathrm{eV}$ and $2.44 \mathrm{eV}(\Delta \mathrm{E}=0.17 \mathrm{eV})$ can be identified. The parallel increase of the PLQE is another proof of the strong reduction of 
interchain species. The PLQE of PDV.Li experiences a fourfold enhancement from the value measured in water, while it increases only by a factor of two in PDV.LiC $\beta$-CD that has an intrinsic low tendency toward aggregation in water.

The suppression or reduction of interchain states induced by alcohols in aqueous solutions of CPEs has been reported by several authors and attributed to interactions of water with the hydrophilic moieties (i.e. charged ionogenic groups) and solvation of the hydrophobic segments (i.e. polymer backbone) by the non-polar chain of alcohols. ${ }^{8,16,24-26}$ Furthermore, our findings relate to previous work $^{27}$ that showed that PDV.Li is highly compatible with non-ionic, non-conjugated polymers such as polyvinyl alcohol (PVA) ${ }^{28}$ and polyethylene oxide $(\mathrm{PEO})^{27}$ and blends of these polymers give homogeneous films with reduced chromophore aggregation and slower exciton diffusion. It was shown that PDV.Li coordinates to PEO in aqueous solutions with a high binding constant and the resulting adducts show single-chain photophysics. The proposed binding mechanism invokes a coordination of the lithium ions by ether groups in $\mathrm{PEO} .{ }^{27}$ It seems likely that propanol molecules are also able to bind to lithium cations through the hydroxyl group, a behavior documented in other simple alcohols, ${ }^{29}$ while the alkyl chain would adsorb on the apolar conjugated backbone of the PDV.Li polymer. These interactions and the hydrophobic/hydrophilic water/alcohol interplay would explain the extraordinary suppression of interchain states formation in the solution containing 50\% propanol.

Interestingly, the PLQE measured in the 1:1 water/propanol mixtures are almost twice higher than that reported for highly diluted solutions in water $\left(10^{-4} \mathrm{mg} \cdot \mathrm{mL}^{-1}\right)$ for both PDV.Li and PDV.LiC $\beta-C D .{ }^{14}$ An improvement of the PLQE is due to a relative faster radiative decay of the excitation compared to the non-radiative processes. However, we do not observe such behavior in our data. In fact, in our experiments, the exciton lifetimes for the 1:1 water/propanol mixtures and those in the highly diluted water solutions are similar. An 
analogous behavior has been interpreted assuming a branching ratio between emissive singlet excitons and the overall absorbing species that include also optically dark interchain species, ${ }^{30,} 31$ and whose formation may be favored when polymer chains are in close proximity. Indeed, we also note that the temporal evolution of the average fluorescence energy features significant differences between the solutions in 1:1 water/propanol and the diluted solutions in water, suggesting that in water, even at low concentrations, polymer chains have a higher tendency to interchain interactions than in the $1: 1$ water/propanol mixture. By comparing our results with the previous report, we observe that the average emission energy in 1:1 water/propanol (Figure S3a) at the excitation time $(\mathrm{t}=0 \mathrm{~ns})$ is about $\sim 50$ and $40 \mathrm{meV}$ higher than in the diluted water solutions, for PDV.Li and PDV.LiC $\beta-C D$ respectively. ${ }^{14}$ We also observe that the spectral diffusion is completed in about $7 \mathrm{~ns}$ in the solutions in alcohol, similarly to the diluted water solutions, but the final energy $(2.47 \mathrm{eV})$ is about $\sim 40 \mathrm{meV}$ higher than in water both for PDV.Li and PDV.LiC $\beta-C D$. This could be due either to a larger torsional distortion of the polymer chain in the alcohol solution, which reduces the effective conjugation length, and/or to a stronger tendency of the polymer chains in water to form strongly coupled interchain species.

\subsection{Aggregation in propanol rich solutions}

The solutions in water/propanol mixtures containing more than $70 \%$ alcohol show more abrupt changes of the photophysical properties by varying the solvent composition. Both PDV.Li and PDV.LiC $\beta-C D$ exhibit a drop of the PLQE by about a third when the propanol fraction is increased from $70 \%$ to $92 \%$, followed by a partial recovery when the solution contain almost exclusively propanol (99.5\%). Despite the similarity of PLQE trends, the other spectroscopic data show distinctive behaviors in the unthreaded and threaded polymer and suggest different responses to the change in solvent composition. 
We attribute the drop in the PLQE to a return of chromophore aggregation at high propanol fraction. However we note that the PLQE in solution with more that $70 \%$ propanol is still relatively higher than that of PDV.Li in pure water suggesting that interchain states formed in propanol have mainly a weaker coupling. We can recognize in the PL spectrum of PDV.Li (Figure 2a) the three vibronic bands observed in the 1:1 water/propanol solution, however we observe a redistribution of intensity from the $0-0$ to the $0-1$ band. This change in relative peaks intensities is consistent with the formation of weakly coupled H-aggregates (see Molecular Modeling and Simulations of Fluorescence Spectra section). The average emission energy (Figure S3a) shows that the excitons emitting in the first ns after the excitation have a high intrachain character $(\mathrm{E} \sim 2.56 \mathrm{eV}$ for $92 \%$ and $95 \%$ and $\mathrm{E} \sim 2.60 \mathrm{eV}$ for $99.5 \%$ propanol content), but from about 2 ns the emission energy rapidly relaxes to values typical of the strongly coupled aggregates with H-like character observed in water solutions $(\mathrm{E} \sim 2.35 \mathrm{eV}) .{ }^{14}$ This suggests that the propanol-rich solutions contain aggregates with various coupling strength and the excitation migrates from weakly to strongly coupled aggregates whose presence is also supported by the simulated PL spectra discussed below.

The reasons for the relative increase of PLQE observed in the 99.5\% propanol PDV.Li solution are not clear, since the PL decay dynamics is very similar to the one of the solution in $95 \%$ propanol. We note that Burrows and co-workers reported a similar trend in PL intensity for an anionic conjugated fluorene-based polyelectrolyte in dioxane-water mixtures with $90-100 \%$ dioxane content. ${ }^{24}$ The high initial emission energy $(\mathrm{E} \sim 2.6 \mathrm{eV})$ of PDV.Li in $99.5 \%$ propanol suggests that aggregated species form with a slower rate than in the solutions in $92 \%$ and $95 \%$ propanol.

The evolution of the photophysical properties of the polyrotaxane solutions is quite different from the one of PDV.Li discussed before. The set of data collected in solutions with high propanol fractions (92\%-99.5\%) is consistent with the emission from both isolated and 
strongly aggregated chains, which should explain the decrease of PLQE observed between solutions containing $70 \%$ and $92 \%$ propanol. The static fluorescence spectra show a broadening, a partial loss of vibronic structure, similar to what observed in the polyrotaxane solution in water, and also a slight redshift $(\sim 10 \mathrm{~nm})$. Furthermore, the strongly non monoexponential and wavelength dependent character of the PL decay (Figure 5b) prove that the polyrotaxane in propanol rich solution undergoes fast exciton migration from intrachain states to strongly aggregated ones. The dynamics of the $0-0$ emission in the first nanoseconds in the $92 \%$ propanol solution accelerates to an apparent lifetime of $360 \mathrm{ps}$, approaching the limit of our experimental resolution (150 ps). The fast exciton migration is also confirmed by the time dependence of the average emission energy (Figure S3b). While the solution in $50 \%$ propanol shows the highest initial $(\sim 2.62 \mathrm{eV})$ and final $(\sim 2.55 \mathrm{eV}$ after $10 \mathrm{~ns})$ average emission energy, the solutions in $92 \%$ and $95 \%$ propanol show a significantly lower initial energy $(\sim 2.52 \mathrm{eV})$ followed to a fast relaxation to an energy of $\sim 2.40 \mathrm{eV}$. The initial and final average energies are close to those observed in the polyrotaxane solution in water, although the relaxation is much slower in the latter. In water, we note a change of slope in the average energy decay curve that has been related to the formation and relaxation of long-lived intrachain species whereas the exciton energy migration happens at ca. 2 ns. ${ }^{14}$ For solutions in $92 \%$ and $95 \%$ propanol, exciton quenching/migration processes may occur on shorter time scale than our experimental resolution (150 ps), which significantly affect the efficiency of the fluorescent emission. At the highest propanol fraction $(99.5 \%)$, the initial emission energy increases to $2.57 \mathrm{eV}$, while showing similar relaxation rate to the $92 \%$ and $95 \%$ propanol solutions. The increase of initial emission energy and PLQE might suggest a reduction the interchain species also indicated by the redistribution of intensity from the $0-1$ to the $0-0$ band. 
Our data show a return of chain aggregation at high propanol fractions in both PDV.Li and its polyrotaxane analogue. This is not surprising as both compounds showed very poor solubility in pure propanol. We therefore infer that an alcohol content between 30 and $70 \%$ in water/1-propanol mixtures best solvates PDV.Li and PDV.LiC $\beta$-CD likely because hydrophobic and hydrophilic interactions are well balanced. Nevertheless we find that the different chemistry at the surface of polymer backbone determines different modality of aggregation in the rotaxinated and unrotaxinated polymer at high propanol fraction. In this regard, a possible explanation for the different behavior of PDV.LiC $\beta-C D$ is that hydrogen bonding between the cyclodextrin rings (possibly mediated by water molecules) leads to a closer packing between the polyrotaxane chains. Indeed, the cyclodextrin solubility in organic solvents and alcohol/water solutions has been reported to decrease due to complex formation. $^{32}$

\subsection{Molecular Modeling and Simulations of Fluorescence Spectra}

A molecular modeling study was performed to complement the detailed spectroscopic investigation. Atomistic simulations of model aggregates provide insight into the interchain packing distances in PDV.Li and PDV.LiC $\beta-C D$ and of the resulting excited-state characteristics. Conclusions drawn from the experimental photophysical investigations are further supported by spectra simulated using phenomenological models of single chains and $\mathrm{H}$-aggregates. The investigation of how solvents distributed around threaded and unthreaded polymer is beyond the scope of this paper.

Optimized ground-state geometries of model aggregates of PDV.Li and PDV.LiC $\beta$ CD are shown in Figure S4. We find close packing of PDV.Li chains at $\sim 3.5-3.6 \AA$ whereas, in PDV.Li $\subset \beta-C D$, the supramolecular encapsulation provided by $\beta$-CD increases this distance to 8.5-9.0 $\AA$. Excited state calculations of these model aggregates (Figure S4a and 
S4b for PDV.Li and PDV.LiC $\beta-C D$ respectively) shed light on the nature of the aggregate excitons. PDV.Li exhibits classical H-aggregate characteristics with the lowest excited state delocalized across multiple chains and the transition dipole moments of individual chains in opposite phases hence cancelling out. The net transition dipole moment and associated oscillator strength $f$ of the aggregate is negligible $(f=0.02)$. In PDV.Li $\subset \beta-C D$, however, the lowest excited state remains localized on a single chain and thus bears the large transition dipole moment and associated oscillator strength of an isolated chain $(f=1.50)$.

Next, we apply phenomenological models ${ }^{17,18,33-35}$ of inter- and intra-chain excitons coupled with intramolecular vibrations to aid in the interpretation of the experimental photophysical data. A description of the Frenkel-Holstein Hamiltonian employed in the spectral simulations has been reported elsewhere, ${ }^{35}$ while full details specific to our simulations of PDV.Li aggregates and parameterisation strategy can be found in the SI. We believe that the spectral evolution as a function of propanol fraction is due to changes in the relative population of three distinct classes of emissive species, namely, i. intrachain exciton states, ii. weakly coupled $\mathrm{H}$-aggregates, iii. strongly coupled $\mathrm{H}$-aggregates with partial intermolecular charge-transfer character.

We focus on the three propanol fractions that give rise to spectra we believe to be most representative of each class of emissive species. We furthermore limit our analysis to the PDV.Li spectra as those of PDV.LiC $\beta-C D$ exhibit similar, yet less pronounced trends thanks to the supramolecular encapsulation afforded by $\beta$-CD. With the highest PLQE and a large 0-0 to 1-0 peak ratio, PDV.Li in 50:50 water:propanol is most representative of emission due to intrachain states. The 5:95 water:propanol mixture features the lowest PLQE at high propanol fraction and a small 0-0 to 1-0 ratio, consistent with weakly coupled aggregates. Finally, the spectra recorded in water are most representative of strongly coupled H-aggregates with partial intermolecular charge-transfer character. 
The vibronic structure of the spectra in 50:50 water:propanol is reasonably well reproduced by a single-mode vibronic progression with a $0-0$ energy of $2.78 \mathrm{eV}$, a vibrational energy of $0.17 \mathrm{eV}$ associated with the $\mathrm{C}=\mathrm{C}$ stretching vibrational mode, a Huang Rhys factor of 1.17 and Gaussian broadening with FWHM $0.18 \mathrm{eV}$ (see Figure 6a). The low energy tail is not captured by the model. This is most likely due to a residual population of strongly coupled interchain states.

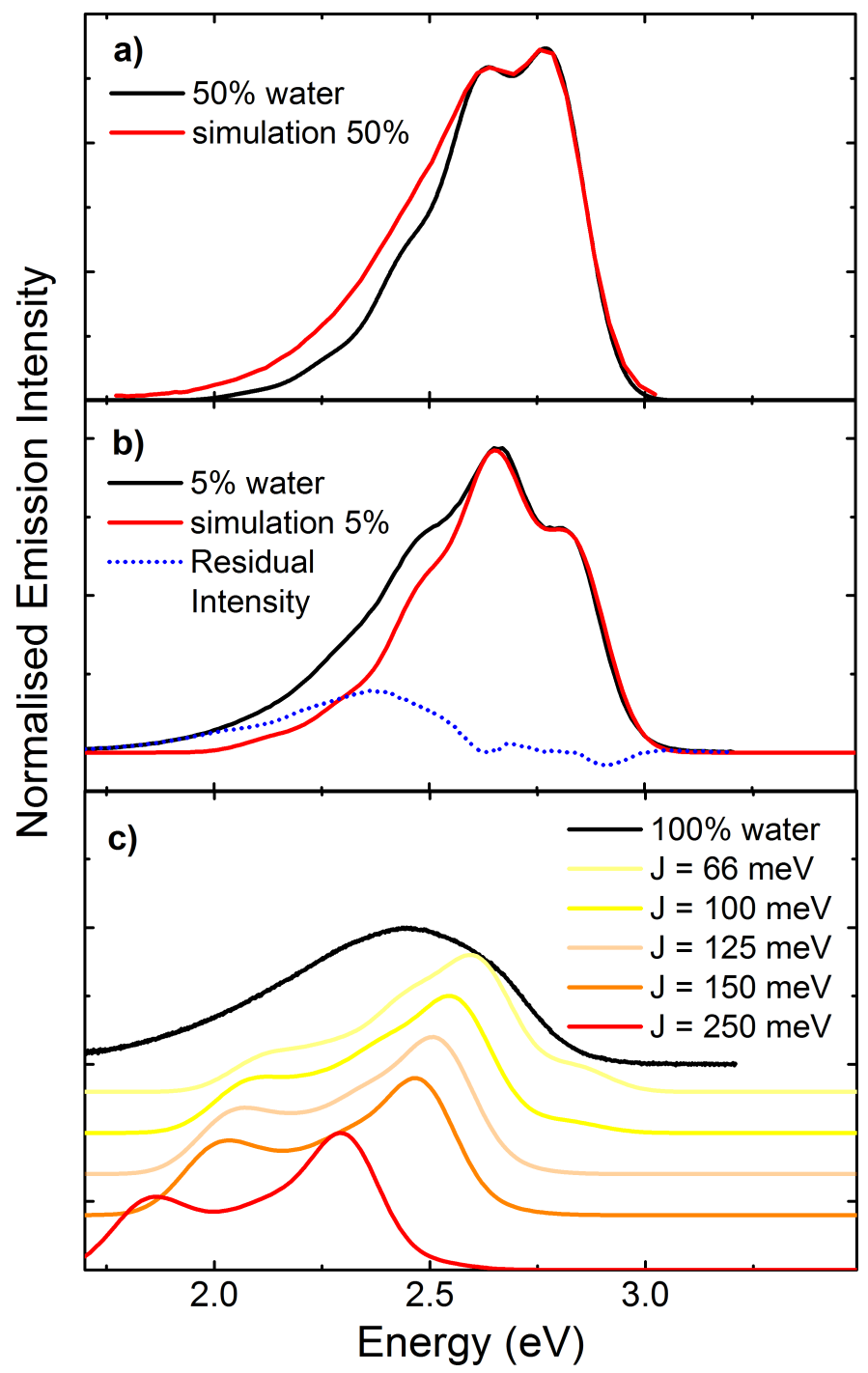

Figure 6 PDV.Li experimental and simulated spectra in water/propanol mixtures from single molecule to aggregate emission. (a), PL spectrum in 50:50 water:propanol (solid black line) and single mode vibronic progression fit (solid red line). (b), PL spectrum in 5:95 water:propanol (solid black line) and simulated aggregate spectrum with an excitonic coupling of $18 \mathrm{meV}$ (solid red line). The residual intensity not accounted for by the model is shown (dotted blue line) and may be due to a small population of strongly coupled aggregates. (c), PL spectrum in water (solid black line) and simulated spectra of strongly coupled Frenkel excitons with varying excitonic coupling $J$ (solid colored lines). The calculated PL spectra are shifted to better visualize the trend. The simulated spectra account for the amount of red shift observed in water, but not for the loss of vibronic structure that it points to a partial charge-transfer character of the emission species of PDV.Li aggregates in water. 
Values of the 0-0 energy, Huang-Rhys factor and vibrational energy associated with the $\mathrm{C}=\mathrm{C}$ stretching vibrational mode derived from the model of 50:50 water:propanol were then used to model the spectra of weakly coupled aggregates observed in 5:95 water:propanol and strongly coupled aggregates. The relative intensities of the $0-0$ and $0-1$ vibronic peaks are reproduced with an excitonic coupling $(J)$ of $18 \mathrm{meV}$ (see Figure 6b). Here also, the model fails to fully account for the low energy tail, which could be explained by a small population of strongly coupled H-aggregates.

Finally, we attempt to simulate the broad featureless and red-shifted spectrum of PDV.Li in water as aggregates comprising strongly coupled Frenkel excitons. Figure 6c demonstrates that excitonic couplings $J$ of $100 \mathrm{meV}$ and larger provide enough red shift yet fail to reproduce the broad structureless emission. While our model takes into account coupling to intramolecular vibrations, it neglects intermolecular vibrations that are expected to couple to emissive states that have a significant share of charge-transfer (CT) excitations. Our model furthermore does not include such CT excitations, providing a description based purely on Frenkel excitations. In cases where emissive states do feature an admixture of Frenkel and CT excitations, large couplings to intermolecular, low-frequency vibrational modes wash out the underlying vibronic structure giving rise to a structureless spectrum such as that of PDV.Li in water (top panel of Figure 2a). The underlying reason for the strong coupling of CT excitations to intermolecular vibrations is a strong decrease in the energy of such configurations at shorter intermolecular distances (see Figure S5), which can be interpreted as being driven mainly by an increase in binding energy as the distance between hole and electron residing on neighboring molecules decreases. It is also worth noting that CT transition energies are also strongly dependent on the local solvation environment, being stabilized by the orientational polarization of polar solvent molecules in their immediate vicinity. The broader and more featureless emission of PDV.Li in solvent mixtures with 
increasing fractions of water is thus consistent with the energetic stabilization of intermolecular CT configurations with higher fractions of water. This could be driven by both a closer intermolecular packing of these hydrophobic molecules as the fraction of water is increased, and the evolution of the first solvation shell from one containing weakly-polar propanol molecules (which might preferentially solvate the hydrophobic PDV.Li even at low concentrations) to one comprising only highly-polar water molecules.

\section{Conclusions}

In conclusion, we have found that mid-range concentrations of 1-propanol $(<70 \%)$ in water significantly reduces the presence of interchain species both in PDV.LiC $\subset$-CD and, to a greater extent, in PDV.Li. In pure water, PDV.Li showed emission from mainly stronglycoupled aggregates with $\mathrm{H}$-like character and partial charge-transfer character. However, when propanol is added to water in a 1:1 ratio, we reported a fourfold increase in the PLQE and emission arising mainly from intrachain states. A similar behavior is shown also for PDV.Li $\subset \beta-C D$ although it appears less striking because in this case the formation of interchain states in water is already effectively prevented by the macrocycle encapsulation. We remark that PLQE values in 1:1 water/propanol mixtures are almost a factor of two higher than reported for highly diluted solutions in water, thereby suggesting that propanol is extremely effective at preventing aggregation also in comparison to "rotaxination" of polymer chains within cyclodextrin rings.

The photophysics of these systems at high propanol content $(>70 \%)$ is more complex and characterized by a different behavior for the threaded and unthreaded polymer. PDV.Li emission properties are consistent with the presence of weakly-coupled aggregates and, to a smaller extent, of strongly-coupled interchain states. Due to the different chemistry at the surface of polymer backbone and possible interactions between the cyclodextrin rings, 
PDV.Li $\subset \beta-C D$ instead forms mainly strongly coupled aggregates. Surprisingly, both the threaded and unthreaded polymers show a reduction of interchain species at very high propanol content $(99.5 \%)$.

\section{Acknowledgements}

This work was supported by the EC Sixth Framework Programme (FP6/2002-2006) under the Grant Agreement No. MRTN-CT-2006-036040 (THREADMILL), the EC Seventh Framework Programme (FP7/2007-2013) under Grant Agreement No. 264694 (GENIUS), and the EU Horizon 2020 Research and Innovation Programme under Grant Agreements No. 643238 (SYNCHRONICS), as well as the Royal Society. FC is a Royal Society Wolfson Research Merit Award holder. The work in Mons has been funded by the Interuniversity Attraction Pole program of the Belgian Federal Science Policy Office (PAI 7/05) and the Belgian National Fund for Scientific Research. DB is a FNRS Research Director. LW acknowledges support from the "Thousand Young Talents Plan" of China and the "Hundred Talents Plan" of Zhejiang University. 


\section{References}

1. F. Cacialli, J. S. Wilson, J. J. Michels, C. Daniel, C. Silva, R. H. Friend, N. Severin, P. Samorì, J. P. Rabe, M. J. O'Connell, P. N. Taylor and H. L. Anderson, Nat Mater, 2002, 1, 160-164.

2. F. Huang, H. Wu and Y. Cao, Chem Soc Rev, 2010, 39, 2500-2521.

3. R. Marcilla, D. Mecerreyes, G. Winroth, S. Brovelli, M. D. R. Yebra and F. Cacialli, Appl Phys Lett, 2010, 96.

4. H. Jiang, P. Taranekar, J. R. Reynolds and K. S. Schanze, Angew Chem Int Ed Engl, 2009, 48, 4300-4316.

5. S. W. Thomas, Joly, G. D. and T. M. Swager, Chem Rev, 2007, 107, 1339-1386.

6. C. Zhu, L. Liu, Q. Yang, F. Lv and S. Wang, Chem Rev, 2012, 112, 4687-4735.

7. C. V. Hoven, A. Garcia, G. C. Bazan and T. Q. Nguyen, Adv Mater 2008, 20, 3793 3810.

8. C. Tan, M. R. Pinto and K. S. Schanze, Chem Commun, 2002, 446-447.

9. M. J. Frampton, T. D. Claridge, G. Latini, S. Brovelli, F. Cacialli and H. L. Anderson, Chem Commun, 2008, 2797-2799.

10. J. J. Michels, M. J. O'Connell, P. N. Taylor, J. S. Wilson, F. Cacialli and H. L. Anderson, Chemistry, 2003, 9, 6167-6176.

11. S. Brovelli, G. Latini, M. J. Frampton, S. O. McDonnell, F. E. Oddy, O. Fenwick, H. L. Anderson and F. Cacialli, Nano Lett, 2008, 8, 4546-4551.

12. A. Farcas, G. Tregnago, A. M. Resmerita, S. T. Dehkordi, S. Cantin, F. Goubard, P. H. Aubert and F. Cacialli, J Polym Sci Pol Chem, 2014, 52, 460-471.

13. F. E. Oddy, S. Brovelli, M. T. Stone, E. J. F. Klotz, F. Cacialli and H. L. Anderson, $J$ Mater Chem, 2009, 19, 2846-2852.

14. A. Petrozza, S. Brovelli, J. J. Michels, H. L. Anderson, R. H. Friend, C. Silva and F. Cacialli, Adv Mater, 2008, 20, 3218-3223.

15. D. R. Delgado and F. Martínez, Phys Chem Liq, 2014, 53, 293-306.

16. C. Y. Tan, M. R. Pinto, M. E. Kose, I. Ghiviriga and K. S. Schanze, Adv Mater, 2004, 16, 1208-1212.

17. J. Clark, C. Silva, R. H. Friend and F. C. Spano, Phys Rev Lett, 2007, 98, 206406.

18. F. C. Spano, J Chem Phys, 2005, 122, 234701.

19. R. Noriega, J. Rivnay, K. Vandewal, F. P. Koch, N. Stingelin, P. Smith, M. F. Toney and A. Salleo, Nat Mater, 2013, 12, 1038-1044.

20. F. C. Spano and C. Silva, Annu Rev Phys Chem, 2014, 65, 477-500.

21. J. Gierschner, H. G. Mack, D. Oelkrug, I. Waldner and H. Rau, Journal of Physical Chemistry A, 2004, 108, 257-263.

22. K. H. Schweikart, M. Hohloch, E. Steinhuber, M. Hanack, L. Luer, J. Gierschner, H. J. Egelhaaf and D. Oelkrug, Synthetic Metals, 2001, 121, 1641-1642.

23. S. Kasiouli, F. Di Stasio, S. O. McDonnell, C. P. Constantinides, H. L. Anderson, F. Cacialli and S. C. Hayes, J Phys Chem B 2013, 117, 5737-5747.

24. H. D. Burrows, S. M. Fonseca, C. L. Silva, A. A. Pais, M. J. Tapia, S. Pradhan and U. Scherf, Phys Chem Chem Phys, 2008, 10, 4420-4428.

25. W. Dou, C. Wang, G. Wang, Q. Ma and X. Su, J Phys Chem B, 2008, 112, 12681 12685.

26. S. Wang and G. C. Bazan, Chem Commun, 2004, 2508-2509.

27. J. S. Wilson, M. J. Frampton, J. J. Michels, L. Sardone, G. Marletta, R. H. Friend, P. Samorì, H. L. Anderson and F. Cacialli, Adv Mater, 2005, 17, 2659-2663.

28. F. Di Stasio, P. Korniychuk, S. Brovelli, P. Uznanski, S. O. McDonnell, G. Winroth, H. L. Anderson, A. Tracz and F. Cacialli, Adv Mater, 2011, 23, 1855-1859. 
29. G. A. Lawrance, M. J. Robertson, Sutrisno and E. I. von Nagy-Felsobuki, Inorg Chim Acta, 2002, 328, 159-168.

30. M. Yan, L. J. Rothberg, F. Papadimitrakopoulos, M. E. Galvin and T. M. Miller, Phys Rev Lett, 1994, 72, 1104-1107.

31. N. T. Harrison, G. R. Hayes, R. T. Phillips and R. H. Friend, Phys Rev Lett, 1996, 77, 1881-1884.

32. K.-H. Frömming and J. Szejtli, Cyclodextrins in pharmacy, Springer Science \& Business Media, 1993.

33. S. T. Salammal, J. Y. Balandier, J. B. Arlin, Y. Olivier, V. Lemaur, L. J. Wang, D. Beljonne, J. Cornil, A. R. Kennedy, Y. H. Geerts and B. Chattopadhyay, J Phys Chem $C, 2014,118,657-669$.

34. M. Kirkus, L. Wang, S. Mothy, D. Beljonne, J. Cornil, R. A. Janssen and S. C. Meskers, J Phys Chem A, 2012, 116, 7927-7936.

35. L. Wang and D. Beljonne, CrystEngComm, 2016, 18, 7297-7304. 


\section{Electronic Supplementary Information}

\section{Increased luminescence efficiency by synergistic exploitation of}

\section{lipo/hydrophilic co-solvency and supramolecular design}

Giulia Tregnago, Michele Serri, Sergio Brovelli, Shane O. McDonnell, Platon Korniychuk, Linjun Wang, Michael Wykes, David Beljonne, Adam Tracz, Harry L. Anderson, Franco Cacialli

\section{Summary}
a. Experimental and computational details
b. Absorption spectra
c. Time-resolved photoluminescence
d. Average emission energy
e. Molecular modeling and simulations

\section{a. Experimental and computational details}

The synthesis and characterization of sulfonate substituted poly(diphenylenevinylene) lithium salt (PDV.Li) and its cyclodextrin polyrotaxane derivative (PDV.LiC $\beta-C D$, with a threading ratio of 2 cyclodextrins per repeat unit) used in this work is described elsewhere ${ }^{1}$.

All data shown were taken for PDV.Li and PDV.LiC $\beta-C D$ solutions in water/propanol mixtures at a concentration of $5 \times 10^{-3} \mathrm{mg} \cdot \mathrm{mL}^{-1}$ and $1.1 \times 10^{-2} \mathrm{mg} \cdot \mathrm{mL}^{-1}$ respectively, in order to account for the different molecular weight and obtain the same optical density. Solutions were prepared from a "master" solution in water, i.e. 1 
$\mathrm{mg} \cdot \mathrm{mL}^{-1}$ and 2.2. $\mathrm{mg} \cdot \mathrm{mL}^{-1}$, for PDV.Li and PDV.LiC $\beta-\mathrm{CD}$ respectively, then diluted 200 times. Therefore, solutions with the highest content of propanol contain $0.5 \%$ of water. Optical absorption spectra were collected in the visible and near-ultraviolet (UV) region by means of a CCD-based spectrophotometer (Agilent 8543) with a spectral resolution of $1 \mathrm{~nm}$. Decay dynamics were studied with a time-correlated single-photon counting (TCSPC) spectrometer using a ps-pulsed diode laser at 371 nm (Edinburgh Instruments EPL-375) and a F-900 TCSPC unit (temporal resolution $\sim 150 \mathrm{ps)}$ with a photomultiplier tube coupled to a monochromator.

The PLQE were measured using a relative method for optical dilute solutions, using as reference the quantum yield of quinine sulfate dehydrate in $1.0 \mathrm{~N}$ sulfuric acid $\left(\sim 10^{-5} \mathrm{M} ; \mathrm{PLQE}=(0.546 \pm 5 \%)\right.$ (NIST standard reference material SRM\# 936a (www.nist.gov/ts/msd/srm/)). Refractive indices for water/1-propanol mixtures (see Table 1) were taken from data reported in the literature. ${ }^{2,3}$

Table S1 Extrapolate refractive indices for water/1-propanol mixtures from references ${ }^{2,3}$

\begin{tabular}{|c|c|}
\hline Water content (\%) & Refractive index \\
\hline 100 & 1.333 \\
\hline 90 & 1.340600734 \\
\hline 80 & 1.348325645 \\
\hline 70 & 1.35499813 \\
\hline 50 & 1.365760976 \\
\hline 30 & 1.375439499 \\
\hline 20 & 1.379749526 \\
\hline 8 & 1.383248019 \\
\hline 5 & 1.383971197 \\
\hline 0.5 & 1.385075773 \\
\hline 0 & 1.3852 \\
\hline
\end{tabular}

All experiments were performed at room temperature and all measurements were corrected for the overall spectral response of the detector. 
Optimized ground-state geometries and excited state calculations of model aggregates of PDV.Li and PDV.LiC $\beta$-CD are shown in Figure S4. Lithium ions are assumed to be dissociated, as is likely the case in water. Each sulfonate group thus adds a charge of -1 to each chain. By adopting a geometry in which neighboring chains are offset with respect to each other along the long polymer axis, the distance between sulfonates is maximized, minimizing the coulomb repulsion between them such that it does not hinder the close packing of PDV.Li chains at $\sim 3.5-3.6 \AA$. In PDV.LiC $\beta-C D$, the supramolecular encapsulation provided by $\beta-C D$ increases this distance to 8.5-9 $\AA$. The ground-state geometry of the PDV.Li model aggregate was optimized at the wB97XD/6-31g(d) level, including a Polarizable Continuum Model (PCM) description of solvent effects using default parameters for water, as implemented in Gaussian09 RevA.02. ${ }^{4}$ As it would have been computationally impractical to perform a similar calculation on the ground-state geometry of PDV.Li $\subset \beta-C D$, semi-empirical method including dispersion and hydrogen bonding corrections for PM6-DH was instead used, as implemented in MOPAC2009. ZINDO/SCI calculations and all simulations of spectra were performed with in-house codes. Details of the precise Frenkel-Holstein model and Hamiltonian employed in the phenomenological spectral simulations have been reported elsewhere. ${ }^{6}$ Briefly, as we are only interested in the low energy part of the optical absorption spectra, we assume that each chromophore in the aggregate has only two electronic states; the ground state $\left(\mathrm{S}_{0}\right)$ and the first excited state $\left(\mathrm{S}_{1}\right)$, which are coupled to an intramolecular symmetric vinyl-stretching mode, and that chromophores within the aggregate interact only with their nearest neighbours, with a constant excitonic coupling term. Our parameterization strategy was as follows; we first simulated an isolated PDV.Li molecule with a single-mode Franck-Condon progression (equivalent 
to the Frenkel-Holstein model with the excitonic coupling set to zero) with up to four vibrational quanta. Hand tuning of the parameters to provide the best agreement with the observed emission spectrum of PDV.Li in 50\% water (believed to arise mainly from single-chain emission) yielded the following experimentally-derived parameters: A $\mathrm{S}_{0}-\mathrm{S}_{1}$ 0-0 transition energy of $2.78 \mathrm{eV}$, an effective vibrational energy of $0.17 \mathrm{eV}$ $\left(\sim 1400 \mathrm{~cm}^{-1}\right)$, a Huang-Rhys factor 1.17 and Gaussian broadening with $\sigma=75 \mathrm{meV}$ (FHWM $\sim 180 \mathrm{meV}$ ). Having fixed these parameters, spectra of model aggregates comprising 10 interacting chromophores were simulated with various values of the excitonic coupling. Simulations made use of the two particle approximation and a maximum vibrational quantum number of four at a temperature of $300 \mathrm{~K}$. No energetic disorder in site energies was employed in order to avoid overfitting of the spectrum with the addition of further parameters.

\section{b. Absorption spectra}

a)

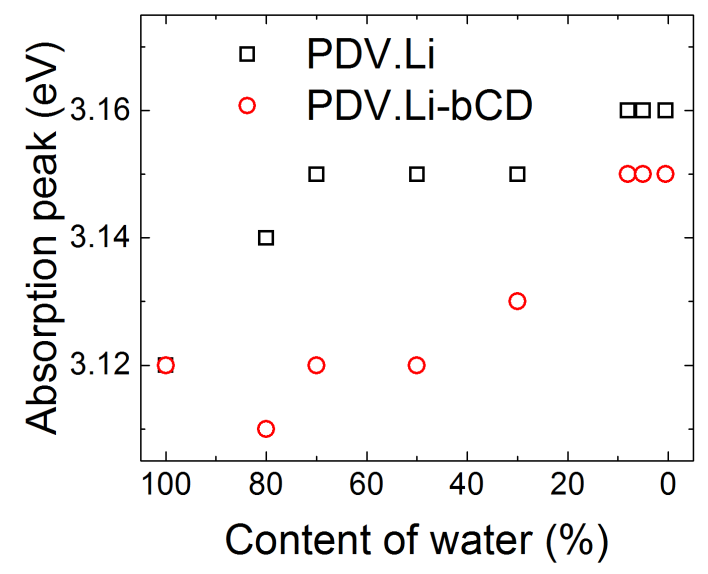

b)

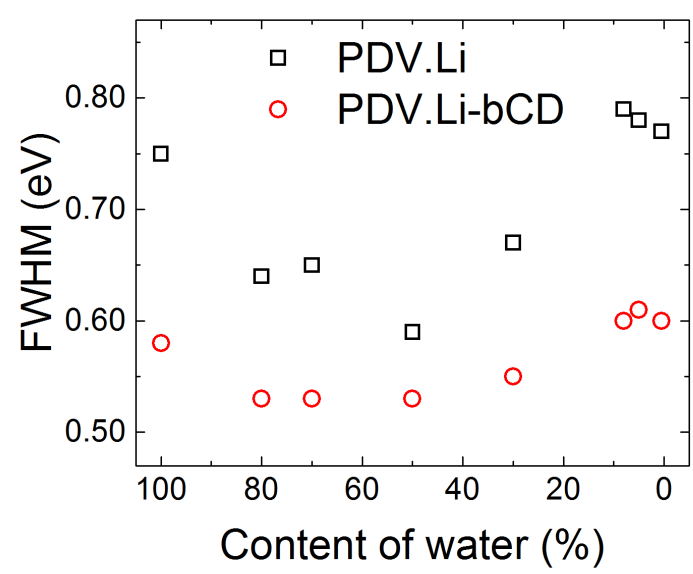

Figure S1 Absorption peak (a) and linewidth (b) for PDV.Li (black squares) and PDV.Lic $\beta$-CD (red circles) in water/1-propanol mixtures as a function of the water content. 


\section{c. Time-resolved photoluminescence}

\section{Figure S2}

a

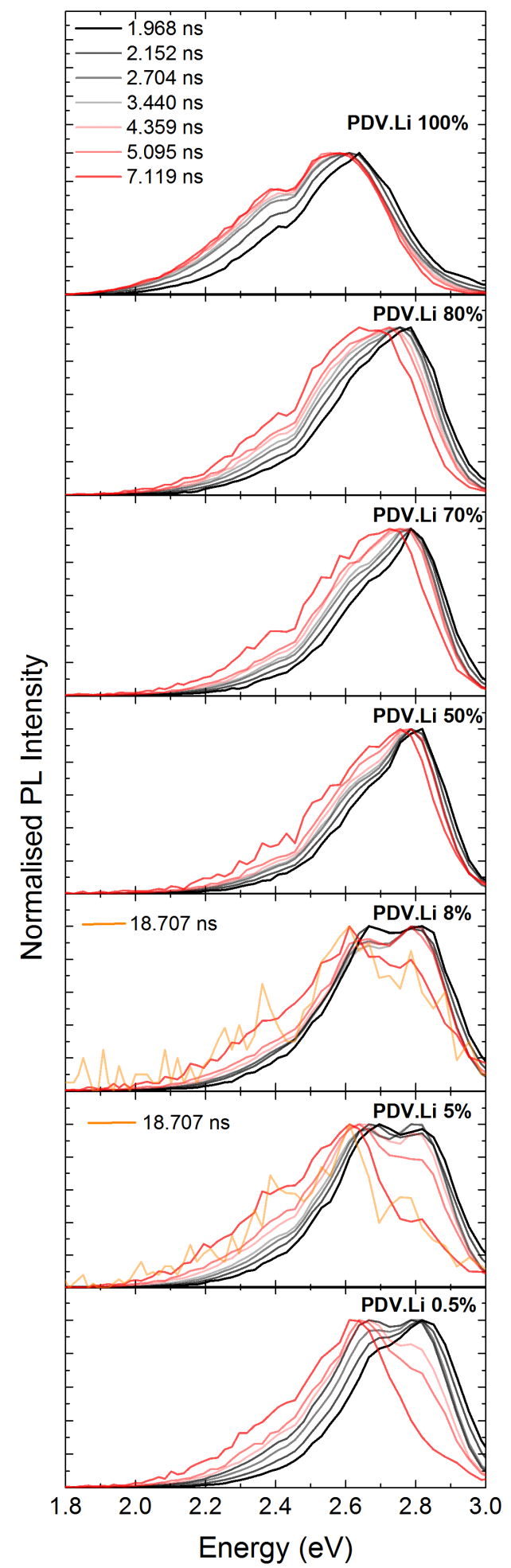

b

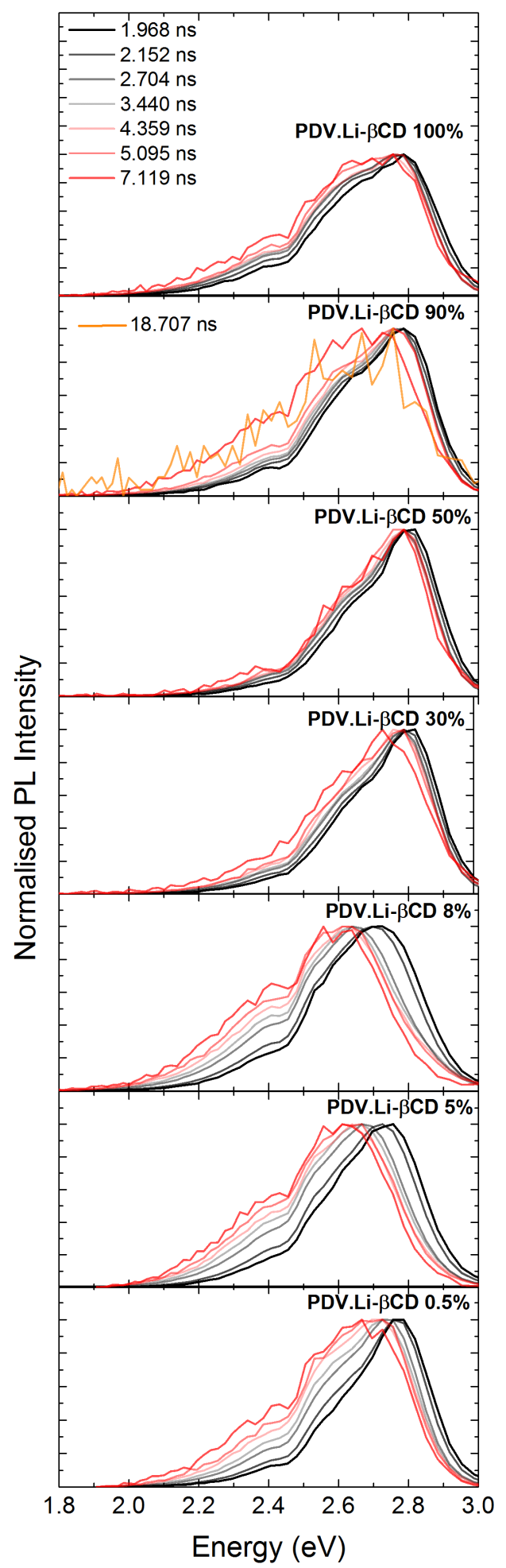

Figure S2 Normalized time-resolved emission spectra of PDV.Li (a) and PDV.LiC $\beta-C D(\mathbf{b})$ aqueous solutions with increasing propanol content ( $\%$ water is reported). The spectra are reported at different times after excitation. 


\section{d. Average emission energy}

Figure S3

a)

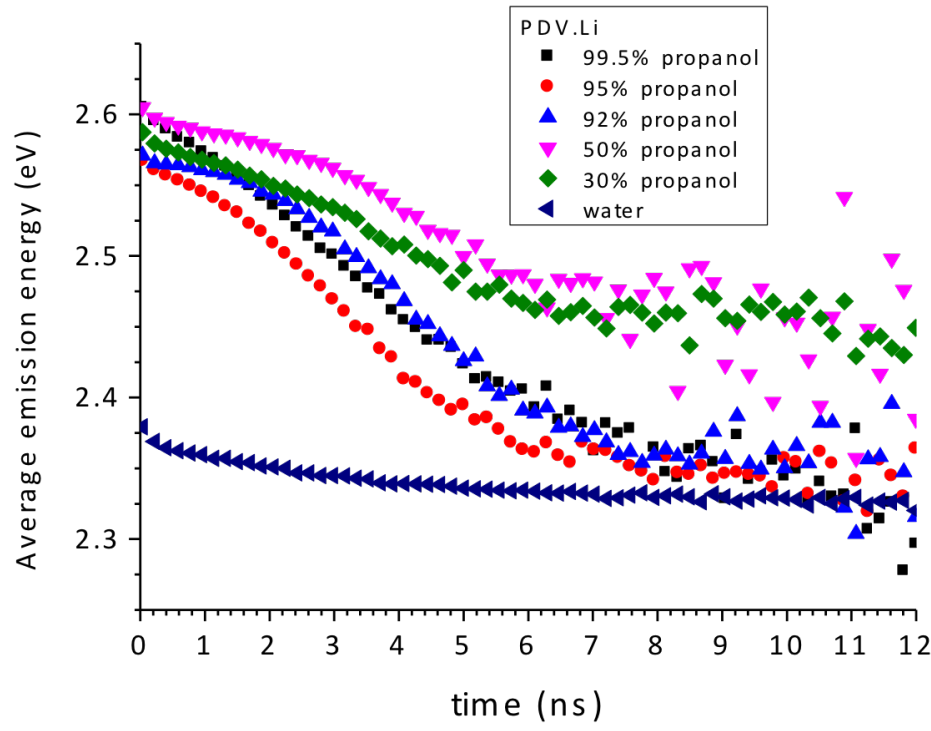

b)

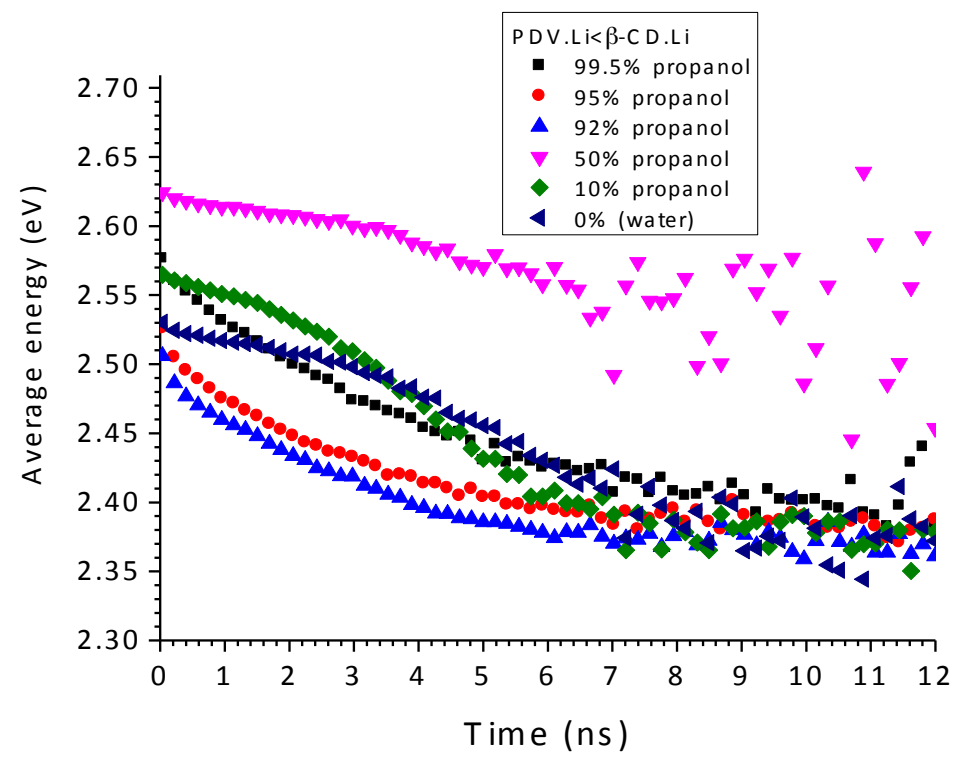

Figure S3 (a) Time dependence of the average emission energy in PDV.Li solutions, (b) Time dependence of the average emission energy in PDV.Li $\subset \beta-C D$ solutions 


\section{e. Molecular modeling and simulations}

a)

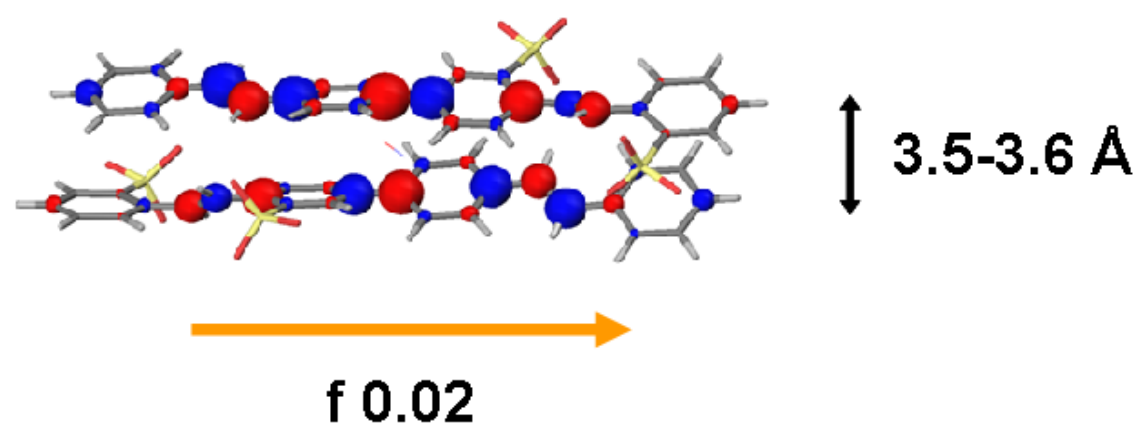

b)
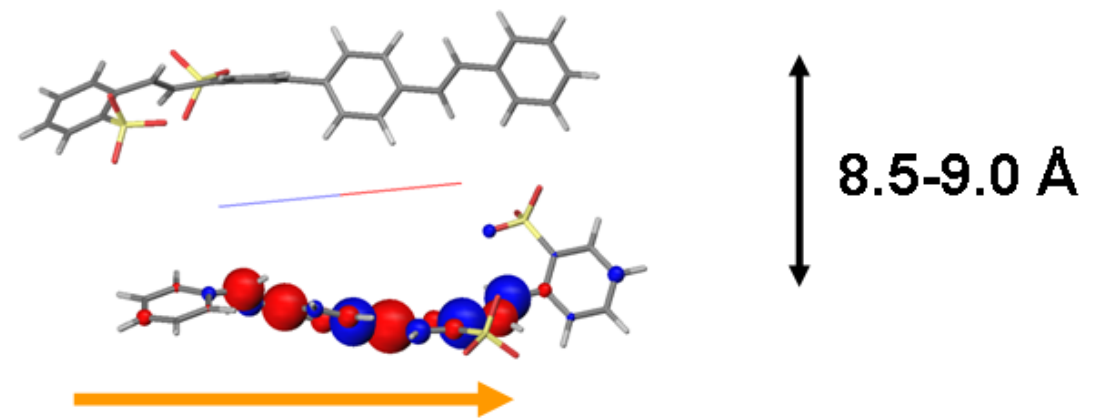

c)

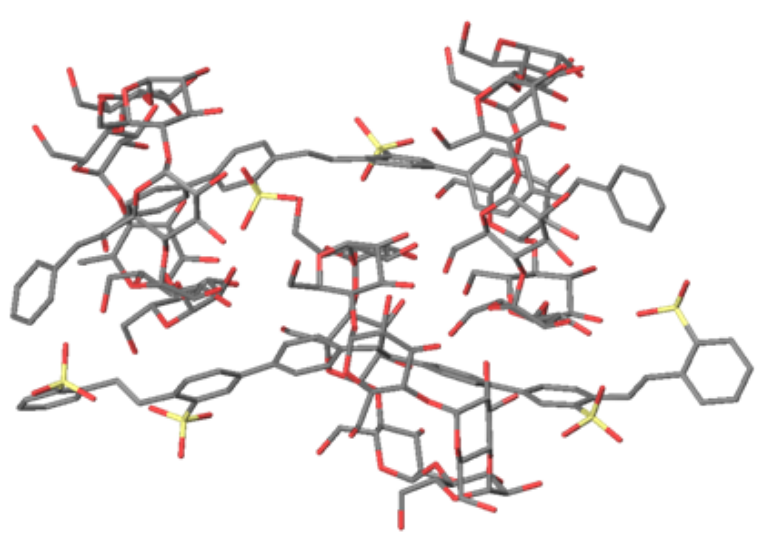

Figure S4 (a and b) Geometries of PDV.Li and PDV.LiC $\beta$-CD model aggregates with ZINDO/CIS atomic transition densities of the lowest excited state shown in blue and red spheres. The interchain distances are $\sim 3.5-3.6$ and 8.5-9 $\AA$, respectively. Orange arrows indicate the direction of the net transition dipole moment of each chain. PDV.Li (a) shows clear H-aggregate characteristics in which transition dipole moments of individual chains cancel resulting in a low oscillator strength $f$, while those of the PDV.LiC $\beta$-CD (b) exhibit characteristics of a single chain with a high oscillator strength. In $\mathbf{b}, \beta-\mathrm{CD}$, was not included in the calculation for clarity and computational efficiency. (c). Geometry PDV.LiC $\beta$-CD model aggregate showing position of $\beta$-CD. Hydrogens have not been drawn for clarity. In all geometries, lithium ions are assumed to be dissociated, as it is likely the case in water. 
The excited state calculations shown in Figure $\mathrm{S} 4 \mathrm{a}$ and $\mathrm{S} 4 \mathrm{~b}$ were performed using ground state geometries and thus capture the extent of the lowest excited state at the time of population prior to any geometric relaxation. In the case of a symmetric cofacial stack in its ground state geometry, one would expect the lowest excited state to be delocalized across chains no matter how large the distance between them. However, for chains at large distances, the reorganization energy will exceed the strength of the interchain excitonic coupling and the exciton will self-localize onto a single chain. The fact that the lowest excited state is created as a localized intrachain exciton suggests the presence of a geometric asymmetry in the PDV.LiC $\beta$-CD stack.

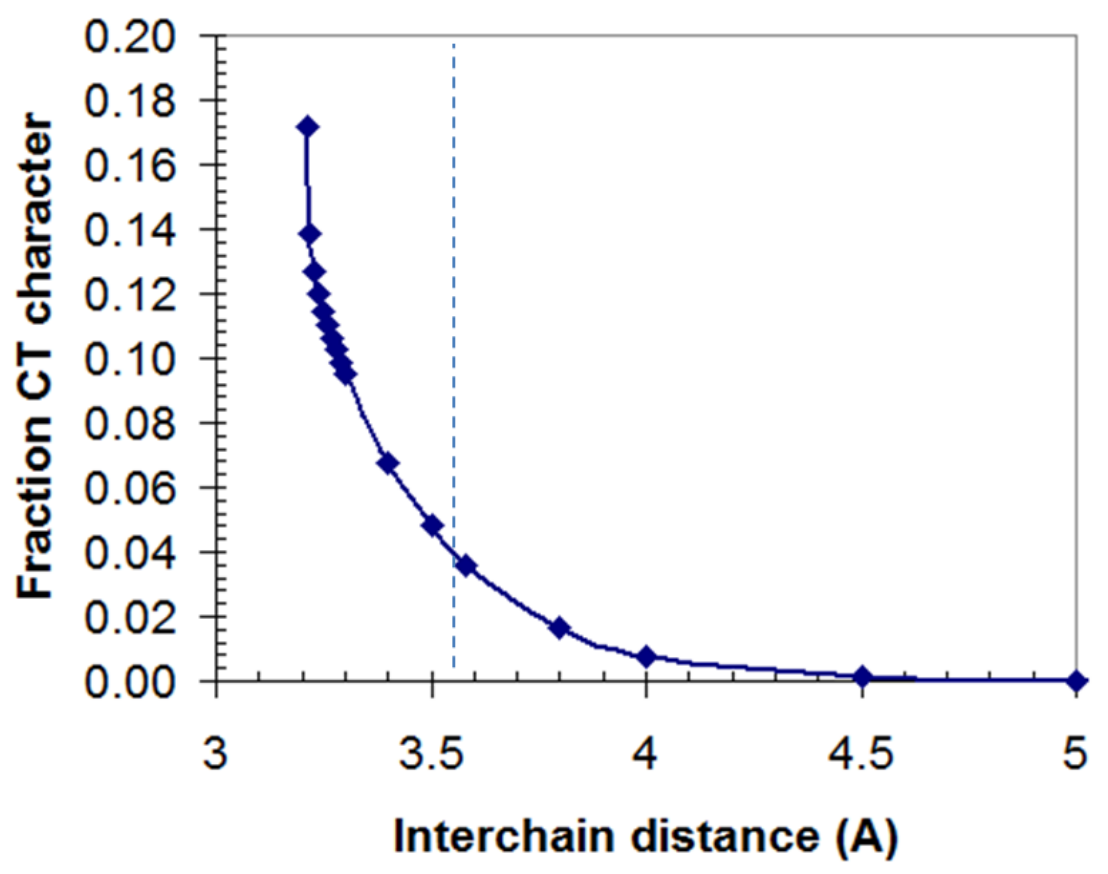

Figure S5 Plot of contribution of charge transfer configurations to the lowest excited state S1 of the model PDV.Li stack as a function of interchain displacement. The dashed line indicates the average stacking distance at the equilibrium geometry of the PDV.Li model aggregate. 


\section{References}

1. J. J. Michels, M. J. O'Connell, P. N. Taylor, J. S. Wilson, F. Cacialli and H. L. Anderson, Chemistry, 2003, 9, 6167-6176.

2. V. I. Kuchuk, I. Y. Shirokova and E. V. Golikova, Glass Physics and Chemistry, 2012, 38, 460-465.

3. K.-Y. Chu and A. R. Thompson, Journal of Chemical and Engineering Data, 1962, 3, 358-360.

4. Frisch, M. J., Trucks, G. W., Schlegel, H. B., Scuseria, G. E., Robb, M. A., Cheeseman, J. R., Scalmani, G., Barone, V., Mennucci, B., Petersson, G. A., Nakatsuji, H., Caricato, M., Li, X., Hratchian, H. P., Izmaylov, A. F., Bloino, J., Zheng, G., Sonnenberg, J. L., Hada, M., Ehara, M., Toyota, K., Fukuda, R., Hasegawa, J., Ishida, M., Nakajima, T., Honda, Y., Kitao, O., Nakai, H., Vreven, T., Montgomery, Jr., J. A., Peralta, J. E., Ogliaro, F., Bearpark, M., Heyd, J. J., Brothers, E., Kudin, K. N., Staroverov, V. N., Kobayashi, R., Normand, J., Raghavachari, K., Rendell, A., Burant, J. C., Iyengar, S. S., Tomasi, J., Cossi, M., Rega, N., Millam, J. M., Klene, M., Knox, J. E., Cross, J. B., Bakken, V., Adamo, C., Jaramillo, J., Gomperts, R., Stratmann, R. E., Yazyev, O., Austin, A. J., Cammi, R., Pomelli, C., Ochterski, J. W., Martin, R. L., Morokuma, K., Zakrzewski, V. G., Voth, G. A., Salvador, P., Dannenberg, J. J., Dapprich, S., Daniels, A. D., Farkas, Ö., Foresman, J. B., Ortiz, J. V., Cioslowski, J., Fox, D. J. Gaussian 09 Rev A.02, Gaussian, Inc., Wallingford CT, 2009.

5. MOPAC: Stewart, James J. P., Stewart Computational Chemistry, Colorado Springs, CO, USA, 2009

6. L. Wang and D. Beljonne, CrystEngComm, 2016, 18, 7297-7304. 